\title{
PROPUESTA DE MEJORAS PARA LA GESTIÓN DE LA INNOVACIÓN EN UNA EMPRESA TECNOLÓGICA
}

\author{
Improvement proposal for the management of innovation \\ in a technology-based company Título en inglés
}

\section{EPISTEMUS}

ISSN: 2007-8196 (electrónico)

ISSN: 2007-4530 (impresa)

\section{Germán Rossetti ${ }^{1}$ \\ Daniela Ferreira ${ }^{2}$ \\ Melisa Donda ${ }^{3}$}

Recibido: 15 de septiembre de 2018 ,

Aceptado: 29 de noviembre de 2018

Autor de Correspondencia:

Ing. Germán Rossetti

Correo:groseti@fiq.unl.edu.ar

\section{Resumen}

El objetivo del presente trabajo es diagnosticar y proponer mejoras para llevar a cabo la gestión de la innovación en una empresa de base tecnológica. Para ello, se aplica una metodología de diagnóstico que permita evaluar la capacidad de una determinada empresa para innovar, debido a que la misma no posee un proceso formal de Gestión de la Innovación Tecnológica. Para alcanzar dicho objetivo, se toma como referencia el estudio de investigación realizado por los autores Quiroga y Borrás (2015). Teniendo en cuenta que, la metodología propuesta por los autores mencionados, consta de siete dimensiones y que para cuantificar cada una de las mismas se deben efectuar una serie de preguntas, es necesario en primer lugar definir la ponderación de dichas preguntas en función de las características de la empresa bajo estudio. Luego, se aplica la metodología para evaluar la capacidad de innovación que posee la empresa. Finalmente, se proponen mejoras a ser implementadas por la empresa con el objetivo de generar condiciones más propicias para la gestión de la innovación.

Palabras clave: Gestión, Innovación, Empresa, Tecnología

\section{Abstract}

The objective of this work is to diagnose and propose improvements to carry out the management of innovation in a technology-based company. For this, a diagnostic methodology is applied that allows evaluating the ability of a certain company to innovate, because it does not have a formal process of Management of Technological Innovation. To achieve this objective, the research study carried out by the authors Quiroga and Borrás (2015) is taken as a reference. Bearing in mind that, the methodology proposed by the aforementioned authors, consists of seven dimensions and that to quantify each one of them, a series of questions must be asked, it is necessary first to define the weighting of said questions according to the characteristics of the company under study. Then, the methodology is applied to evaluate the innovation capacity that the company possesses. Finally, improvements are proposed to be implemented by the company with the aim of generating more favorable conditions for the management of innovation.

Keywords: Management, Innovation, Company, Technology.

Fac. de Ing. Qca., UN del Litoral. Sgo del Estero 2829, Sta Fe, Argentina. groseti@fiq.unl.edu.ar 1 Fac. de Ing. Qca., UN del Litoral. Sgo del Estero 2829, Sta Fe, Argentina. ferreiracamposdaniela@gmail.com 2 Fac. de Ing. Qca., UN del Litoral. Sgo del Estero 2829, Sta Fe, Argentina. melidonda@hotmail.com 3 


\section{INTRODUCCIÓN}

En la actualidad las empresas están inmersas en un mundo cada vez más globalizado, donde la competencia es mayor, lo que implica el creciente interés de ellas por innovar, desarrollar y mejorar sus productos o servicios para ocupar una posición de liderazgo en el mercado. Por lo tanto, es fundamental estar a la vanguardia de las necesidades actuales y utilizar ciertas herramientas que ayuden a ofrecer mejores productos o servicios, y que permitan obtener mayores beneficios, tanto económicos como sociales, tecnológicos, de prestigio, entre otros (Fernández Sánchez, 2005; Freitas-Filho, 2013). La innovación tecnológica es un proceso donde se introducen en el sistema productivo nuevas combinaciones de factores de producción que permiten disponer de un nuevo producto o producir uno ya existente a un menor costo. También se considera que la innovación es tecnológica cuando se relaciona con la ciencia y la tecnología (Escorsa y Valls, 2005; Nowacki y Bachnik, 2016; Krušinskas y Benetytè, 2015; Cavdar y Aydin, 2015; Quiroga y Rossetti, 2016).

Los autores Quiroga y Borrás (2015), llevaron a cabo una investigación en donde desarrollaron una metodología de diagnóstico que permite evaluar la capacidad que poseen las empresas de base tecnológicas para innovar, y en función de dicho diagnóstico proponen determinadas acciones para mejorar la gestión de la innovación. La metodología desarrollada, básicamente constituye una estructura teórica, que consta de siete dimensiones, las cuales fueron corroboradas en empresas industriales, localizadas en Sorocaba, Brasil. Para valorar cada una de las dimensiones se efectúan una serie de preguntas cuantificables.
El objetivo del presente trabajo es diagnosticar y proponer mejoras para llevar a cabo la gestión de la innovación en una empresa de base tecnológica. Para ello, se aplica una metodología de diagnóstico que permita evaluar la capacidad de una determinada empresa para innovar, debido a que la misma no posee un proceso formal de Gestión de la Innovación Tecnológica. Para alcanzar dicho objetivo, se toma como referencia el estudio de investigación realizado por los autores Quiroga y Borrás (2015). Teniendo en cuenta que, la metodología propuesta por los autores mencionados, consta de siete dimensiones y que para cuantificar cada una de las mismas se deben efectuar una serie de preguntas, es necesario en primer lugar definir la ponderación de dichas preguntas en función de las características de la empresa bajo estudio. Luego, se aplica la metodología para evaluar la capacidad de innovación que posee la empresa. Finalmente, se proponen mejoras a ser implementadas por la empresa con el objetivo de generar condiciones más propicias para la gestión de la innovación.

Descripción de la metodología de diagnóstico para la gestión de la innovación en empresas de base tecnológica

Según Quiroga y Borrás (2015), las dimensiones propuestas son una síntesis de las buenas prácticas del sector industrial estudiado en la Región Metropolitana de Sorocaba, y son útiles para apoyar el proceso de gestión de la innovación tecnológica, y ayudan a la elaboración de un diagnóstico confiable sobre la forma en que estos sectores industriales manejan la innovación tecnológica. A lo largo del tiempo, la implementación de estas dimensiones puede influir en las normas culturales de las empresas, y

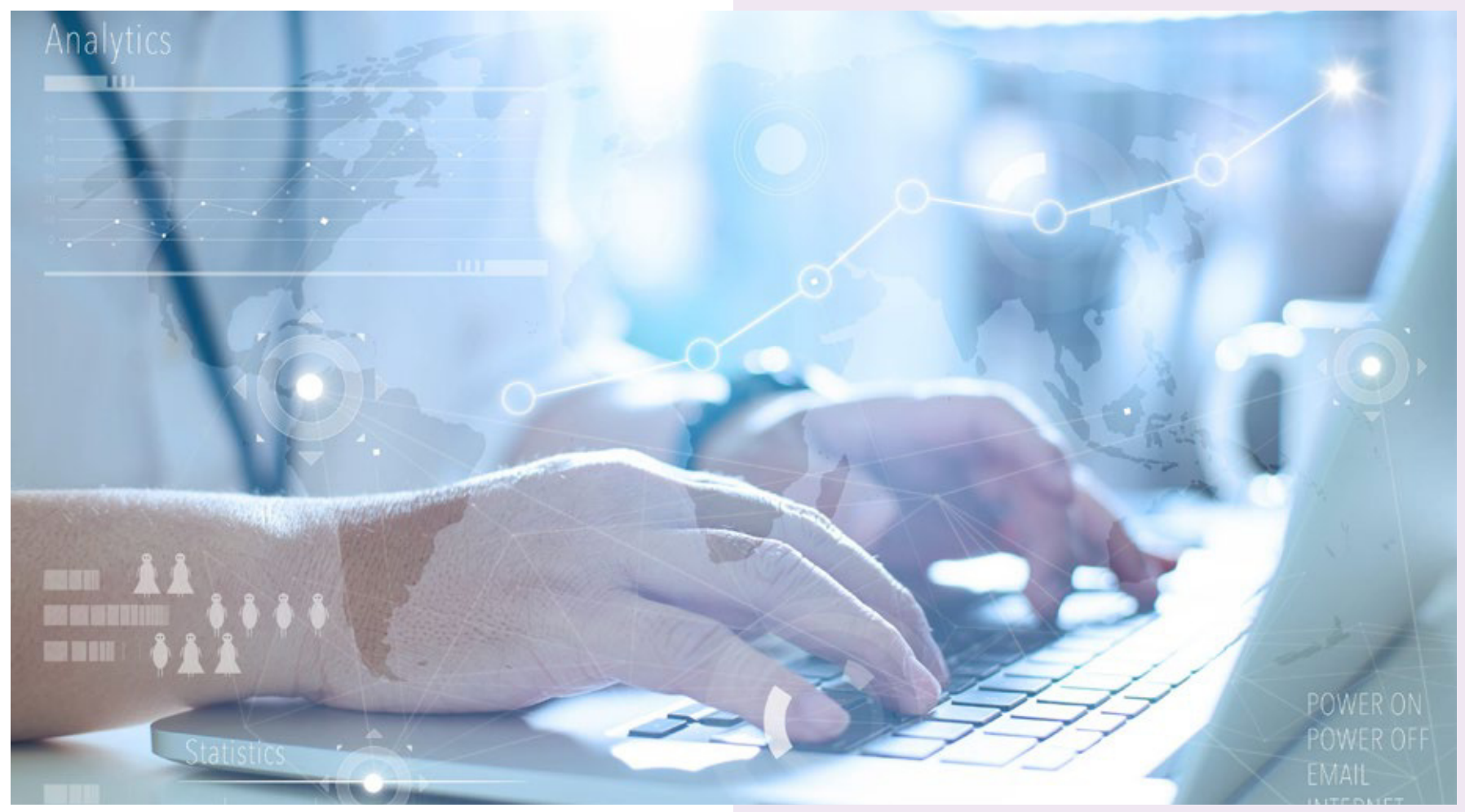




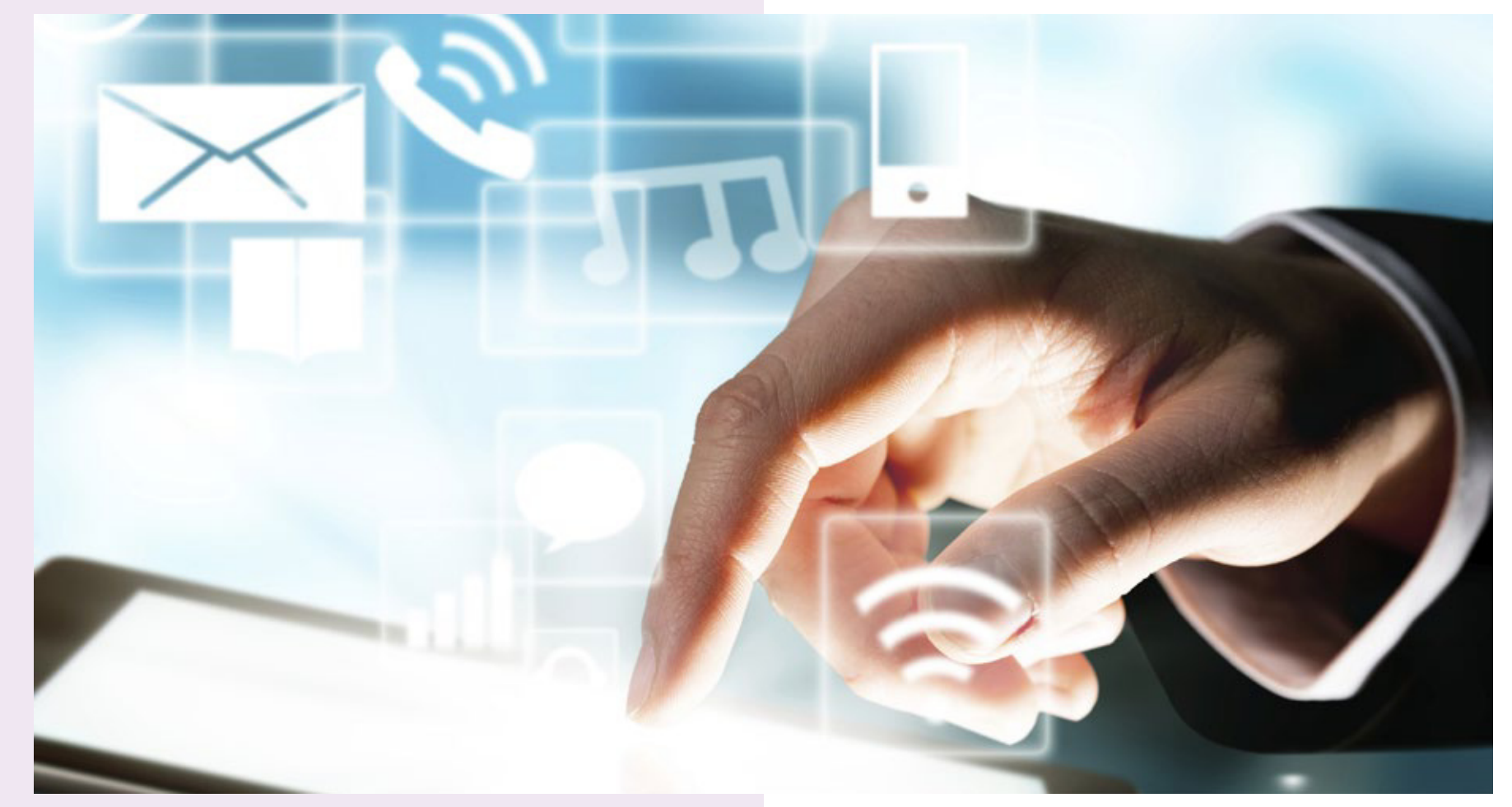

contribuir así al desarrollo de un ambiente para la gestión eficaz de la innovación tecnológica. Para comprender la metodología de diagnóstico de la gestión de la innovación propuesta por los autores mencionados, a continuación se describen las siete dimensiones involucradas:

1. Desarrollo del Proceso de Innovación: Esta práctica de estudio es muy relevante porque la mayoría de las empresas no logran detectar los procesos de innovación que se están llevando a cabo, por lo tanto tampoco se detectan controles ni registros de esas innovaciones. Además, muchas organizaciones desconocen el concepto correcto de innovación, y no cuentan con un sistema de gestión de la innovación tecnológica, o disponen de uno muy simple, con un Departamento de I+D muy acotado, que no incorpora una oficina de patentes y registros de los procesos innovativos.

2. Indicadores y Resultados de Ia Innovación: Esta dimensión plantea la importancia de analizar la conducta tecnológica de las organizaciones, medir sus esfuerzos innovativos, y evaluar y comparar los efectos logrados. Para ello, es sumamente importante el uso de indicadores de rendimiento de la innovación tecnológica. Por lo tanto, se propone el uso de indicadores de desempeño de innovación, que sean monitoreados periódicamente, siendo conveniente para tal fin la utilización de sistemas informáticos. Los indicadores propuestos deben estar vinculados con los objetivos de la empresa, así como también, relacionados con las actividades de innovación.

3. Difusión: En esta dimensión se analizan las relaciones entre las empresas con los sistemas de educación regional y/o nacional, o entre las compañías con las agencias gubernamentales de I\&D, así como también con parques tecnológicos. Por otro lado, se analiza la relación con los proveedores, así como también la participación de los clientes en la innovación de procesos y/o productos.

4. Organización y Estructura Innovadora: En este punto se analiza si la estructura organizacional de la empresa favorece la gestión de la innovación tecnológica. El cambio tecnológico amenaza siempre el orden existente en la organización, ya que muchas empresas no se adaptan con suficiente rapidez a estos cambios debido a la rigidez de sus estructuras organizacionales. Entonces, se proponen indicadores para diagnosticar si la estructura de la organización favorece la innovación, el espíritu emprendedor y el surgimiento de nuevas ideas.

5. Aprendizaje y Capacidad Tecnológica: Este eje aborda el aprendizaje tecnológico como un proceso que involucra varios mecanismos que capturan el conocimiento tecnológico a partir de fuentes internas y externas a la empresa, a fin de transformarlos en recursos tecnológicos de la empresa. En base a su capacidad tecnológica, la empresa consigue no sólo producir bienes y servicios, sino también innovar, lo que significa perfeccionar los productos, procesos de producción, procesos gerenciales existentes o crear nuevos productos, procesos de producción, servicios y procedimientos organizacionales inéditos. Se proponen indicadores vinculados al aprendizaje, de manera de detectar la existencia de inversión para el desarrollo de los empleados, capacidad de la empresa para captar sus habilidades, y aprovechar las lecciones aprendidas.

6. Proyectos de Desarrollo de Productos: Esta dimensión analiza la existencia de una planificación del ciclo de 
vida de los productos, la realización de investigaciones sistemáticas de nuevos productos o procesos y finalmente, como es la evolución de los proyectos de nuevos productos.

\section{Estrategia General, Innovación y Tecnología: Este} punto es trascendente para detectar cómo las empresas gestionan la innovación tecnológica. Normalmente, las empresas poseen una planificación estratégica global o planificación general. Sin embargo, es habitual que no tengan una planificación estratégica de la innovación ni de la tecnología, pero en ciertas ocasiones las empresas cuentan con alguno de ellos, los cuales no están bien integrados con la planificación estratégica.

Para cada una de las siete dimensiones estudiadas, se propone una serie de preguntas, que conforman el cuestionario del Diagnóstico Evaluador de la Capacidad de una Empresa para Innovar, el cual es aplicado en una PyME de base tecnológica, radicada en la Provincia de Santa Fe, Argentina.

\section{Descripción de la empresa}

La empresa bajo estudio pertenece al grupo de pequeñas y medianas empresas (PyMEs), según la Secretaría de la Pequeña y Mediana Empresa y Desarrollo Regional, a través de la resolución $N^{\circ} 21 / 2010$. Está ubicada en la región centro de la Provincia de Santa Fe, y se dedica a la planificación y ejecución de trabajos de automatismo, electricidad industrial, electrificación rural y equipamientos especiales para la industria alimenticia. También, comercializa insumos eléctricos y electrónicos para la industria en general. En el área de automatización y control, la organización brinda soluciones de alto nivel técnico y tecnológico. Se logra la ejecución de proyectos que permiten a los clientes controlar y manejar sus empresas. La empresa se adapta a la medida de las necesidades del cliente, brindando ingeniería y desarrollo continuo para una solución adecuada a cada solicitud. En la Figura 1 se puede visualizar el organigrama actual de la compañía.

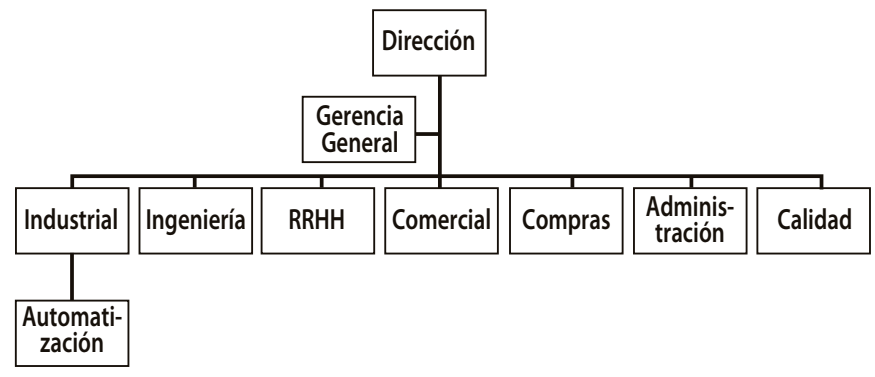

Figura 1. Organigrama Actual.

Fuente: Elaboración propia

Los principales clientes de la organización bajo análisis pertenecen al sector alimenticio de la región, destacándose las industrias lácteas, que buscan a través de una solución innovadora, un aumento significativo en su eficiencia de producción, de manera de lograr una ventaja competitiva, haciendo un mejor uso de los recursos laborales y mejorando el control de calidad. Cabe destacar, que la actividad láctea es sumamente importante en la provincia de Santa Fe, Argentina, por lo que es fundamental que las empresas de este sector gestionen adecuadamente sus procesos aplicando métodos y técnicas específicas.

\section{Diagnóstico de la situación actual de la empresa}

Para realizar el diagnóstico de la empresa, en relación a cómo lleva a cabo el proceso de Gestión de la Innovación, se han utilizado técnicas de relevamiento de información consistentes en encuestas semi-estructuradas y observación in situ. Se han entrevistado a treinta empleados y directivos que conforman la compañía. En función de la información recabada a través del procedimiento mencionado, se enumeran los principales aspectos críticos detectados: (i) se observa una ausencia de estrategias de implementación de nuevas tecnologías y falta de técnicas de búsqueda de nuevas tecnologías, (ii) se evidencia la falta de un proceso de innovación tecnológico definido y se descarta la utilización de indicadores de innovación, sumado a un desconocimiento por parte de los empleados del concepto de innovación tecnológica. Estos inconvenientes repercuten negativamente en el desarrollo del proceso de innovación.

A raíz de los aspectos críticos mencionados anteriormente, se decide aplicar la metodología de diagnóstico propuesta por Borrás y Quiroga (2015) en la organización bajo análisis, con la finalidad de evaluar la capacidad de la misma para innovar, y así poder proponer alternativas de mejoras para la gestión de la innovación.

Aplicación de la metodología de diagnóstico de la gestión de la innovación en la empresa bajo estudio

Para aplicar la metodología de diagnóstico, presentada por Borrás y Quiroga (2015), es necesario ponderar todas las respuestas que componen cada dimensión. Por lo tanto, se propone la utilización de una escala de puntuación de cada pregunta del uno al diez, donde se considera al valor uno como "muy malo", y al diez como "excelente", contemplando también la opción de "no sabe/no contesta" en los casos en que el empleado no tenga conocimiento acerca de la pregunta en cuestión. El cuestionario correspondiente a la metodología se realiza a los treinta empleados que posee la empresa.

Al procesar los resultados obtenidos es necesario realizar un promedio ponderado de las respuestas por dimensión, siendo la sumatoria igual a uno. En cada dimensión, se establecen distintas ponderaciones para cada respuesta, teniendo en cuenta los conocimientos, la participación, la antigüedad y el sector de cada empleado en la empresa. De esta manera, se obtiene un promedio ponderado para cada una de las siete dimensiones que conforman el diagnóstico. El criterio que se utiliza para considerar que cada una de las preguntas de las dimensiones que forman parte de la metodología de 
de desempeño bien alineado con los principales objetivos de la organización. También debe implementar un proceso de innovación óptimo y formal con un sistema de indicadores de desempeño que permita medir e identificar cuándo y dónde se puede mejorar la gestión de la innovación tecnológica. Otra propuesta que debe analizar la empresa es implementar un sistema para monitorear su participación en el mercado e implementar una metodología que le permita disminuir los tiempos de introducción de nuevos productos y procesos en el mercado, así como desarrollar métodos que le posibiliten incrementar la velocidad de innovación. En la Tabla 2 se pueden observar las preguntas de la presente dimensión, con sus respectivos promedios ponderados.

Tabla 2. Preguntas y Promedios Ponderados de la Dimensión 2.

\begin{tabular}{|c|c|c|}
\hline Dimensión & Preguntas & $\begin{array}{l}\text { Promedio } \\
\text { Ponderado }\end{array}$ \\
\hline \multirow{7}{*}{2} & $\begin{array}{l}\text { ¿La empresa utiliza indicadores } \\
\text { tradicionales de: costo, financieros } \\
\text { y calidad, así como de: lanzamiento } \\
\text { de proyectos, actividades } \\
\text { de innovación, desempeño } \\
\text { económico? }\end{array}$ & 6,78 \\
\hline & $\begin{array}{l}\text { ¿Los indicadores son monitoreados } \\
\text { usando sistemas informáticos? ¿Los } \\
\text { planes de acción son generados y } \\
\text { ejecutados siempre que cualquier } \\
\text { indicador no esté de acuerdo con } \\
\text { lo esperado? }\end{array}$ & 7,81 \\
\hline & $\begin{array}{l}\text { ¿Los indicadores de desempeño } \\
\text { están alineados con los objetivos } \\
\text { de la organización e incentivan el } \\
\text { comportamiento deseado? }\end{array}$ & 4,20 \\
\hline & $\begin{array}{l}\text { ¿La empresa usa indicadores para } \\
\text { medir e identificar dónde y cuándo } \\
\text { se puede mejorar la gestión de la } \\
\text { innovación tecnológica? }\end{array}$ & 2,40 \\
\hline & $\begin{array}{l}\text { ¿La empresa tiene un monitoreo } \\
\text { constante de su participación } \\
\text { en el mercado? ¿Son realizadas } \\
\text { evaluaciones de tendencias en } \\
\text { el lanzamiento de los nuevos } \\
\text { productos? }\end{array}$ & 4,98 \\
\hline & $\begin{array}{l}\text { ¿La empresa usa indicadores para } \\
\text { medir cuál es la proporción de } \\
\text { las ventas provenientes de los } \\
\text { productos de innovación y el grado } \\
\text { de innovación de los procesos y } \\
\text { productos? }\end{array}$ & 6,37 \\
\hline & $\begin{array}{l}\text { ¿El tiempo de introducción de } \\
\text { nuevos productos y procesos es } \\
\text { corto? ¿La velocidad de innovación } \\
\text { de la empresa es elevada? }\end{array}$ & 5,95 \\
\hline
\end{tabular}

Fuente: Elaboración propia
Dimensión 3: "Difusión". En este caso, la fórmula que se utiliza para el cálculo de los promedios ponderados nuevamente el peso más alto se asigna a Gerencia General $(0,4)$. La segunda ponderación más elevada $(0,3)$ se atribuye al Área Comercial, incluyendo a todo el personal dentro de la misma, debido a la estrecha relación que se establece con los clientes, donde la difusión es una estrategia fundamental en la Gestión de la Innovación. También se utiliza este peso para el Área Compras/RRHH como consecuencia del contacto directo con los proveedores, quienes también están involucrados activamente en el proceso de Innovación. Para el personal de los Departamentos Industrial e Ingeniería y sus respectivos Gerentes de Área, se toma un peso de 0,2; por ser los representantes de la empresa ante el cliente a la hora de ejecutar los proyectos in situ y por permanecer largos períodos de tiempo en la empresa del cliente, captando de forma continua las necesidades y expectativas del mismo. La menor puntuación $(0,1)$ se da en los Departamentos de Administración y Calidad. El primero, a pesar de que tiene una relación de tipo económica y financiera con los clientes y proveedores, no establece un vínculo directo para la difusión de la Innovación de la empresa. El segundo, por su parte, no establece interacción alguna con los mismos. Para el caso de esta dimensión, las preguntas que están por debajo del límite aceptado son la número 12,16 y 17 . Se plantea a la empresa disponer de un proceso estructurado de análisis de necesidades de los clientes y de comunicación para todos los empleados, definiendo claramente los alcances, las metas y los indicadores. Como así también, desarrollar una estrategia de innovación abierta e identificar necesidades claves y dónde encontrarlas, desarrollar mecanismos para formar y gestionar relaciones de intercambio de conocimiento. Otra solución que se propone es implementar un proceso bien estructurado de generación de nuevas ideas, donde se consideren las necesidades de los clientes, y en el que puedan participar todos los empleados. En la Tabla 3 se pueden observar las preguntas de la dimensión 3 con sus respectivos promedios ponderados.

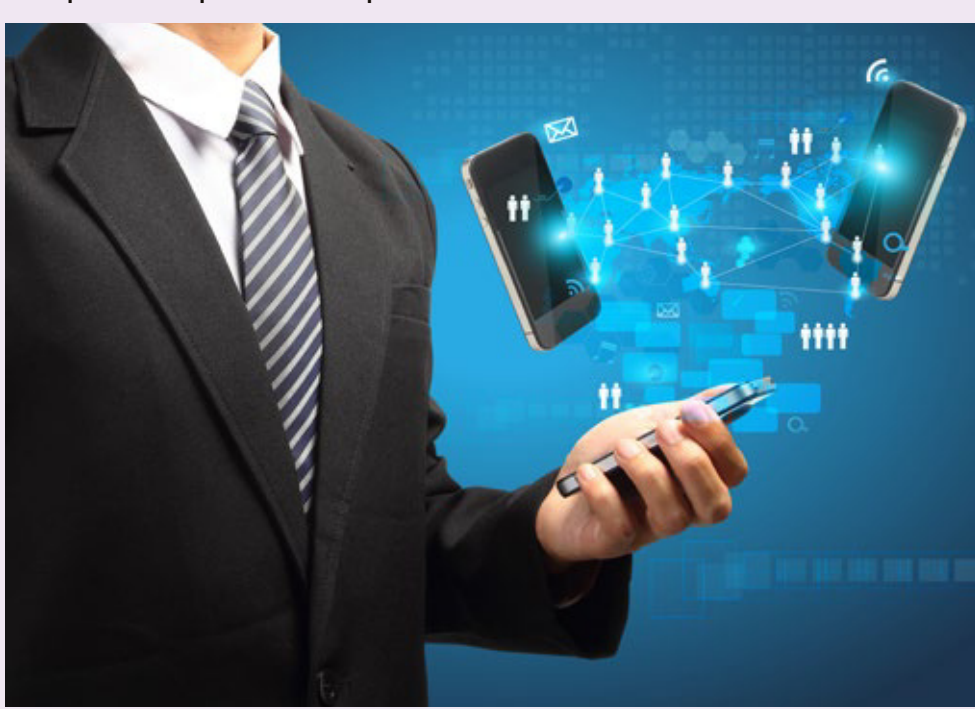


Tabla 3. Preguntas y Promedios Ponderados de la Dimensión 3.

\begin{tabular}{|c|c|c|}
\hline Dimensión & Preguntas & $\begin{array}{l}\text { Promedio } \\
\text { Ponderado }\end{array}$ \\
\hline \multirow{8}{*}{3} & $\begin{array}{l}\text { ¿Las necesidades de los clientes son } \\
\text { analizadas y comunicadas a todos los } \\
\text { empleados? }\end{array}$ & 5,79 \\
\hline & $\begin{array}{l}\text { ¿La empresa tiene buena relación con } \\
\text { sus proveedores y clientes, y estos } \\
\text { están involucrados en el proceso de } \\
\text { innovación? }\end{array}$ & 7,26 \\
\hline & $\begin{array}{l}\text { ¿La empresa trabaja junto a sus } \\
\text { clientesyproveedores enlabúsqueda } \\
\text { y desarrollo de nuevos procesos/ } \\
\text { productos? }\end{array}$ & 7,70 \\
\hline & $\begin{array}{l}\text { ¿Se forman alianzas de colaboración } \\
\text { con otras empresas para desarrollar } \\
\text { nuevos productos o procesos para } \\
\text { beneficio mutuo? }\end{array}$ & 6,46 \\
\hline & $\begin{array}{l}\text { ¿La empresa trabaja con } \\
\text { universidades/centros de educación } \\
\text { técnica/parques } \\
\text { que ayuden a desarrollar su } \\
\text { conocimiento? }\end{array}$ & 2,29 \\
\hline & $\begin{array}{l}\text { ¿Hay un proceso formal de generación } \\
\text { de nuevas ideas basado en el } \\
\text { entendimiento de las necesidades de } \\
\text { los clientes y en donde los empleados } \\
\text { participen? }\end{array}$ & 4,49 \\
\hline & $\begin{array}{l}\text { ¿Son evaluadas las alternativas de } \\
\text { desarrollo de nuevas tecnologías con } \\
\text { otras empresas? }\end{array}$ & 7,02 \\
\hline & $\begin{array}{l}\text { ¿La empresa trabaja próxima al } \\
\text { sistema de educación local y nacional } \\
\text { para comunicar las necesidades de } \\
\text { habilidades técnicas y conocimiento } \\
\text { especializado? }\end{array}$ & 6,32 \\
\hline
\end{tabular}

Fuente: Elaboración propia

Dimensión 4: “Organización y Estructura Innovadora". Para esta dimensión, para el cálculo de los promedios ponderados para las ocho preguntas referentes a este eje, se utiliza una ponderación de 0,4 para el Gerente General de la organización, de 0,35 para los Gerentes de Área y de 0,25 para el resto del personal de la empresa. En este caso se considera que los pesos son relativamente cercanos entre sí, debido a que la opinión de todos los empleados es relevante en el análisis de la estructura organizacional. En esta dimensión solo la pregunta número 25 se encuentra por debajo del límite aceptado, por lo que se propone revisar y desarrollar un sistema de reconocimiento y recompensas relacionado con la innovación, promoviendo la equidad entre todos los miembros de los equipos de proyecto. En la Tabla 4 se puede observar las preguntas de esta dimensión, con sus respectivos promedios ponderados.
Tabla 4. Preguntas y Promedios Ponderados de la Dimensión 4.

\begin{tabular}{|c|c|}
\hline Dimensión & Preguntas \\
\hline
\end{tabular}

¿La estructura de la organización favorece la ocurrencia de la innovación, y permite el surgimiento de emprendedores internos?

¿Las personas de distintos departamentos trabajan bien en conjunto? ¿La organización provee apoyo en término de tiempos, autonomía y recompensas?

¿Los empleados están involucrados con la gestión de las ideas para la mejora de los productos o procesos? ¿Las fallas/errores son tolerados y no sancionados?

¿La estructura organizacional permite tomar decisiones ágiles y rápidas, mientras la gerencia general tiene el compromiso de asumir los riesgos en la innovación de productos y procesos?

7,18

¿La comunicación entre los miembros del equipo y entre los equipos de proyecto funciona eficazmente de arriba hacia abajo, de abajo hacia arriba y a través de la organización?

¿El sistema de recompensa y reconocimiento apoya la innovación $y$ es bien equitativo con todos los miembros de los equipos de proyecto?

¿La empresa tiene un clima de apoyo para nuevas ideas, donde el conocimiento es incentivado y recompensado?

¿En la empresa se trabaja en equipos de proyecto flexible y ágil?

Fuente: Elaboración propia

Dimensión 5: "Aprendizaje y Capacidad Tecnológica". En la fórmula que se utiliza para calcular las medias ponderadas de las respuestas para las siete preguntas de esta dimensión, se plantea la asignación de un peso de 0,6 para las respuestas del Gerente General de la empresa, ya que en él recaen las decisiones referentes al entrenamiento y desarrollo de personas, a la adquisición de aprendizaje tecnológico y a la transformación de este aprendizaje en capacidades tecnológicas de la organización. Para el resto de los empleados y personal jerárquico de mando medio se establece una ponderación de 0,4; teniendo en cuenta que sus opiniones son igualmente prioritarias para todos los sectores y niveles, y por lo tanto de gran importancia para la evaluación de esta dimensión. En este caso únicamente 
la pregunta número 30 no es aceptada, por lo que se plantea la implementación de comentarios post-proyectos y otros métodos de captura de aprendizaje. Además, se propone introducir un sistema con las descripciones de las habilidades individuales de cada uno de los miembros de los equipos de proyecto. En la Tabla 5 se observan las preguntas de esta dimensión 5 , con sus promedios ponderados.

Tabla 5. Preguntas y Promedios Ponderados de la Dimensión 5.

\begin{tabular}{|c|c|c|}
\hline Dimensión & Preguntas & $\begin{array}{l}\text { Promedio } \\
\text { Ponderado }\end{array}$ \\
\hline \multirow{7}{*}{5} & $\begin{array}{l}\text { ¿La empresa se compromete e invierte } \\
\text { en el entrenamiento y desarrollo de las } \\
\text { personas en todos los niveles? }\end{array}$ & 6,86 \\
\hline & $\begin{array}{l}\text { ¿La empresa forma alianzas con otras } \\
\text { organizaciones/empresas y comparte } \\
\text { experiencias que la ayudan a aprender } \\
\text { para beneficio mutuo? }\end{array}$ & 6,57 \\
\hline & $\begin{array}{l}\text { ¿Las habilidades individuales son } \\
\text { efectivamente aprovechadas dentro y } \\
\text { entre los equipos de proyecto? }\end{array}$ & 5,98 \\
\hline & $\begin{array}{l}\text { ¿La empresa se toma el tiempo para } \\
\text { revisar sus proyectos y aprender de sus } \\
\text { errores para mejorar su desempeño? }\end{array}$ & 7,49 \\
\hline & $\begin{array}{l}\text { ¿La empresa considera al aprendizaje } \\
\text { tecnológico como un proceso de } \\
\text { adquisición de conocimientos } \\
\text { tecnológicos de fuentes internas } \\
\text { y externas a la empresa, para } \\
\text { transformarlos en capacidades } \\
\text { tecnológicas de la propia empresa? }\end{array}$ & 7,62 \\
\hline & $\begin{array}{l}\text { En base al conocimiento tecnológico } \\
\text { de la empresa, ¿Se considera la } \\
\text { capacidad tecnológica como un } \\
\text { conjunto de recursos? }\end{array}$ & 7,76 \\
\hline & $\begin{array}{l}\text { ¿El proceso de innovación ocurre en } \\
\text { base a las capacidades tecnológicas } \\
\text { que están distribuidas externamente a } \\
\text { la propia empresa? }\end{array}$ & 6,11 \\
\hline
\end{tabular}

Fuente: Elaboración propia

Dimensión 6: "Proyectos de Desarrollo de Productos". En la fórmula para el cálculo de los promedios ponderados de las respuestas de las siete preguntas incluidas en esta dimensión, la mayor ponderación $(0,5)$ se aplica al Gerente General de la empresa, quien lleva a cabo la gestión de los diversos proyectos con una visión más amplia del funcionamiento general de la organización y de las distintas unidades funcionales que la componen. De igual manera, se asigna un peso elevado $(0,4)$ a las respuestas de los Gerentes de Área de Industrial, Ingeniería y Comercial, ya que éstos junto al Gerente General, conforman un equipo de Programación y Control de la Producción, que tiene como objetivo el diseño de los proyectos de desarrollo de productos en un horizonte de planificación determinado. El resto de los integrantes de los diversos sectores de la empresa tiene una puntuación menor $(0,1)$, debido a que no están involucrados directamente con el desarrollo de los productos y su planificación, y por lo tanto sus respuestas no contribuyen demasiado al resultado final.

Para el caso de esta dimensión, las preguntas que se hallan por debajo del límite aceptado son la número 35, 39 y 40 . Se propone incorporar un sistema de planificación del ciclo de vida de los productos orientado por el mercado; implementar diferentes procesos estructurados que permitan integrar todo el ciclo de vida de los productos, incluyendo el descarte final después de su vida útil. La empresa debe desarrollar una estrategia bien clara de innovación de productos y/o procesos vinculada al análisis de la cartera; implementar un procedimiento sistemático de desarrollo de ideas de nuevos productos y/o procesos, así como también implementar métodos de búsqueda estructurados como benchmarking, ingeniería inversa, etc. En la Tabla 6 se pueden observar las preguntas de esta dimensión, con sus respectivos promedios ponderados.

Tabla 6. Preguntas y Promedios Ponderados de la Dimensión 6.

\begin{tabular}{l|l|c|} 
Dimensión & \multicolumn{1}{|c|}{ Preguntas } & $\begin{array}{c}\text { Promedio } \\
\text { Ponderado }\end{array}$ \\
\hline & $\begin{array}{l}\text { ¿La empresa tiene una planificación } \\
\text { del ciclo de vida de los productos } \\
\text { orientados por el mercado? }\end{array}$ & 5,17 \\
$\begin{array}{l}\text { ¿La empresa usa una metodología } \\
\text { de ingeniería de proyectos como } \\
\text { un proceso estructurado para el } \\
\text { desarrollo de nuevos productos? }\end{array}$ & \multirow{2}{*}{7,55} \\
$\begin{array}{l}\text { ¿Los empleados tienen autonomía } \\
\text { y capacidad para la resolución de } \\
\text { problemas y utilizan eficazmente } \\
\text { herramientas y metodologías de } \\
\text { gestión? }\end{array}$ & \\
$\begin{array}{l}\text { ¿El uso de software de simulación, } \\
\text { modelado 3D, prototipado, y de } \\
\text { sistemas de información está } \\
\text { presente en el desarrollo de } \\
\text { proyectos? }\end{array}$ & \\
\hline
\end{tabular}

¿La empresa utiliza procesos estructurados que integran todo el ciclo de vida del producto, incluyendo el descarte final después de su vida útil?

¿La empresa investiga sistemáticamente ideas de nuevos productos y procesos?

¿Existe una flexibilidad adecuada en el sistema de desarrollo de productos de la empresa que permite que aparezcan pequeños 7,57 proyectos de corta duración?

Fuente: Elaboración propia 


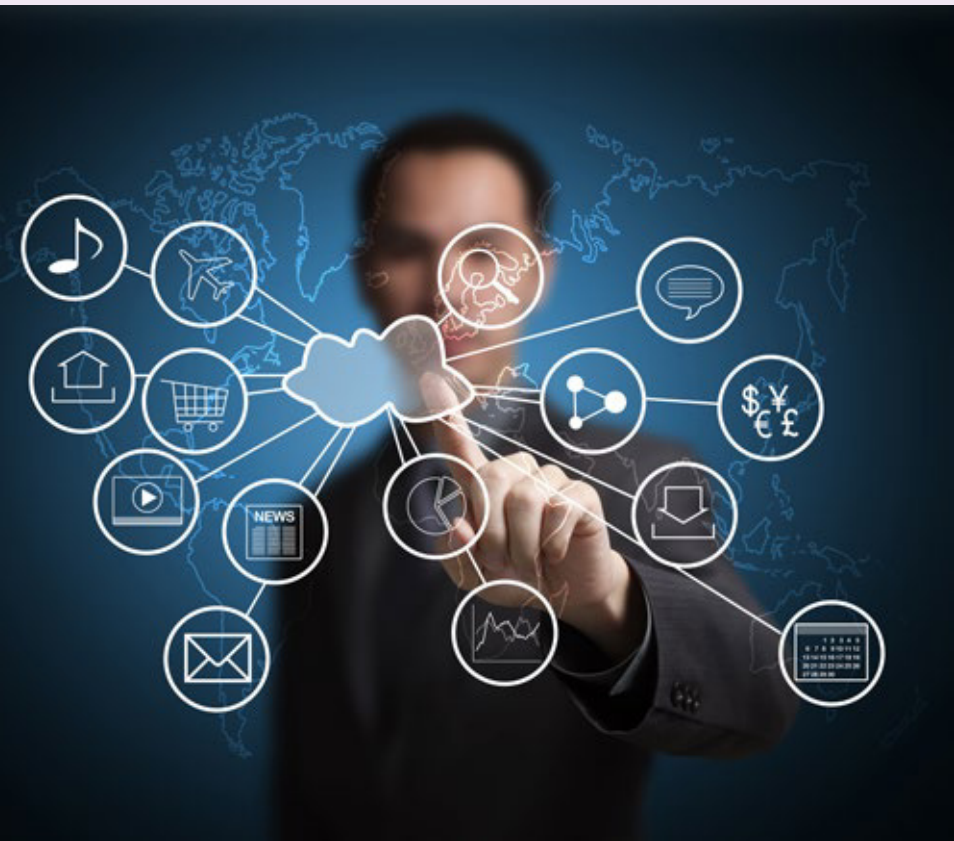

Dimensión 7: “Estrategia General, Innovación y Tecnología". Para concluir con el análisis, en la fórmula que se utiliza para calcular los resultados finales de las nueve preguntas que se incluyen en la presente dimensión, se asigna una puntuación de 0,5 para el Gerente General de la empresa en estudio, quien establece las distintas estrategias a cumplir dentro de la organización en relación a Innovación y Tecnología. Los Gerentes de Área tienen una puntuación alta para sus respuestas $(0,4)$ porque cooperan en la definición y el seguimiento de las estrategias establecidas por la Gerencia General, con el fin de alcanzar los objetivos fijados por la organización. Por este motivo, sus respuestas tienen una fuerte relevancia en los resultados del análisis. Una ponderación mucho menor $(0,1)$ se aplica al resto del personal de la empresa, ya que no tienen participación significativa en la formulación y monitoreo de la estrategia general, de Innovación y de Tecnología.

Este caso es de particular interés ya que todos los resultados se hallan por debajo del mínimo establecido. Para alcanzar la mejora, la empresa debe implementar un plan estratégico de innovación y de tecnología, ambos alineados con el plan estratégico general, y deben ser comunicados a todos los empleados. También debe desarrollar un proceso de planificación estratégica claro, relacionando la innovación con otros proyectos; implementar un procedimiento de búsqueda de patentes y benchmarking, que posibilite el monitoreo de nuevas tecnologías. Para alcanzar una mejor posición en cuanto a la estrategia general de innovación y tecnología, la empresa debe implementar un plan estratégico, incluyendo un programa de innovación y tecnología con un fuerte impulso y enfocado a largo plazo. En la Tabla 7 se pueden observar las preguntas de la dimensión 7 , con sus respectivos promedios ponderados.
Tabla 7. Preguntas y Promedios Ponderados de la Dimensión 7.

\begin{tabular}{|c|c|c|}
\hline Dimensión & Preguntas & $\begin{array}{l}\text { Promedio } \\
\text { Ponderado }\end{array}$ \\
\hline \multirow{9}{*}{7} & $\begin{array}{l}\text { ¿La estrategia de innovación está } \\
\text { claramente definida, es comunicada a } \\
\text { todos los empleados y es usada para } \\
\text { establecer otras estrategias? }\end{array}$ & 4,46 \\
\hline & $\begin{array}{l}\text { ¿La estrategia de tecnología está } \\
\text { claramente definida, todos los } \\
\text { empleados la conocen y ésta es usada } \\
\text { para establecer otras estrategias? }\end{array}$ & 5,65 \\
\hline & $\begin{array}{l}\text { ¿Sehacensistemáticamentebúsquedas } \\
\text { de patentes y benchmarking, como } \\
\text { así también el monitoreo continuo de } \\
\text { nuevas tecnologías? }\end{array}$ & 3,95 \\
\hline & $\begin{array}{l}\text { ¿Existen estrategias deimplementación } \\
\text { de nuevas tecnologías de proceso que } \\
\text { puedan mejorar los niveles de calidad, } \\
\text { costoy servicio post-venta, e incorporar } \\
\text { nuevas ventajas competitivas? }\end{array}$ & 4,56 \\
\hline & $\begin{array}{l}\text { ¿Las estrategias de innovación y } \\
\text { tecnología son flexibles y permiten } \\
\text { responder a los cambios del mercado? }\end{array}$ & 3,80 \\
\hline & $\begin{array}{l}\text { ¿Existe una planificación eficaz e } \\
\text { integrada de innovación y tecnología, } \\
\text { incluyendo análisis técnico, legal y de } \\
\text { impacto económico y ambiental? }\end{array}$ & 2,77 \\
\hline & $\begin{array}{l}\text { ¿Existe una fuerte relación entre los } \\
\text { proyectos de innovación tecnológica } \\
\text { y la estrategia general de la empresa? }\end{array}$ & 4,27 \\
\hline & $\begin{array}{l}\text { ¿Existe compromiso y apoyo de la } \\
\text { alta dirección para la innovación, } \\
\text { asumiendo los riesgos en el desarrollo } \\
\text { de nuevos productos/procesos? }\end{array}$ & 5,96 \\
\hline & $\begin{array}{l}\text { ¿El programa de innovación } \\
\text { tecnológica tiene un enfoque a largo } \\
\text { plazo? }\end{array}$ & 3,73 \\
\hline
\end{tabular}

Fuente: Elaboración propia

\section{RESULTADO DEL DIAGNÓSTICO}

En cuanto al resultado global del diagnóstico, que se obtiene mediante el promedio de todas las respuestas de todos las dimensiones, se concluye que la empresa tiene un resultado de 5,97; lo que refleja una dificultad importante para la compañía, ya que impide que ésta pueda desarrollar sus ventajas competitivas y alcanzar el éxito en su gestión innovadora, teniendo en cuenta que es de base tecnológica y el mayor porcentaje de sus proyectos incluyen productos y procesos innovadores. Por otra parte, cabe aclarar que las respuestas procesadas, cuyos promedios ponderados se encuentran dentro del rango aceptado, deben ser también consideradas al momento de plantear estrategias para mejorar, es decir, se busca que los resultados sean mayores o iguales al actual a lo largo del tiempo, ya que este proceso es continuo y persigue 
un mejor posicionamiento de la empresa, de manera que refleje una exitosa gestión de la innovación tecnológica.

Teniendo en cuenta que la Innovación Tecnológica es un proceso donde permanentemente se introducen en el sistema productivo nuevas ideas, que permiten disponer de nuevos productos y/o procesos, es importante lograr y sostener la continuidad de este proceso innovador para alcanzar el desarrollo de nuevas ventajas competitivas de la empresa. Para lograr la innovación continua se parte de una combinación de experiencias adquiridas, valores e información, que tiene origen y es aplicada por los integrantes de la organización, no sólo a partir de documentos de información, sino también de las rutinas de trabajo y de las prácticas de gestión. Esto está directamente relacionado con la capacidad que tiene la empresa de acceder a información, generar nuevas ideas y transformarlas en conocimiento, diseminándolo por toda la organización e incorporándolo en los productos y procesos. Por lo tanto, es de suma importancia una medición puntual y objetiva, como la aplicada mediante los cuestionarios descriptos anteriormente en este caso de estudio, a todos los integrantes de la empresa, para la realización de un diagnóstico preciso que indique la capacidad de innovación de la misma. En caso de corresponder la aplicación de medidas correctivas, la metodología de diagnóstico desarrollada incluye para cada tópico de estudio las propuestas de mejora a aplicar en cada pregunta con resultado no aceptado.

Para poder llevar a cabo las mejoras correspondientes, luego de la aplicación de los cuestionarios, es necesario un periodo de tiempo considerable para evaluar su repercusión en la organización hasta la próxima medición. El plazo que se supone óptimo para la implementación de la metodología de diagnóstico planteada corresponde a un año, en una primera etapa. Luego, cuando se alcance el grado deseado de innovación será factible realizar un análisis más prolongado en el tiempo, que queda a juicio del evaluador.

\section{CONCLUSIONES}

El objetivo del presente trabajo fue diagnosticar y proponer mejoras para llevar a cabo la gestión de la innovación en una empresa de base tecnológica. A partir del estudio llevado a cabo, se puede concluir:

El resultado global que se obtiene al aplicar la metodología de diagnóstico $(5,97)$ refleja que la compañía no está desarrollando adecuadamente sus ventajas competitivas, y que posee un bajo grado de innovación.

El diagnóstico y la propuesta de mejoras proporcionada a la empresa es el punto de partida para garantizar una gestión exitosa y continua. La metodología de diagnóstico es una herramienta que permite medir la capacidad de la empresa para innovar, e incluye propuestas de mejora, las cuales ayudan a la organización a alcanzar una mayor presencia en el mercado, destacándose con procesos y productos tecnológicos de alto impacto. Éstos incluyen también servicios tecnológicos que brinda la empresa, y que acompañan al cliente en todas las etapas del ciclo de vida del producto.

Teniendo en cuenta los aspectos críticos que se detectaron en la organización, y las mejoras que deben implementarse para solucionarlos, se sugiere la incorporación de un área dentro del organigrama de la empresa denominada "Gestión de la Innovación Tecnológica", cuyo objetivo principal sea definir las estrategias de innovación y de tecnología; fijar un método de registro de información y exigir su cumplimiento; gestionar búsquedas de patentes y benchmarking; impulsar la puesta en valor de proyectos innovadores; y brindar capacitación y motivación a todos los miembros de la organización.

La incorporación de un área que gestione formalmente la innovación es esencial en este tipo de empresas de base tecnológica, para asegurar una eficiente gestión ya que sus procesos y productos tecnológicos se tornan cada vez más competitivos, por su alto grado de innovación y calidad.

\section{AGRADECIMIENTOS}

Los autores agradecen la contribución económica brindada por la Agencia Nacional de Promoción Científica y Tecnológica, a través del Fondo para la Investigación Científica y Tecnológica (PICT 2015 - No 1629) y a la UNL (CAI+D 2016 PI 50120150100216LI).

\section{BIBILIOGRAFÍA}

[1] Cavdar, S.C. y Aydin, A. D. (2015). An Empirical Analysis about Technological Development and Innovation Indicators. Procedia-Social and Behavioral Science, 195, 1486-1495.

[2] Escorsa, P. y Valls, J. (2005). Tecnología e innovación en la empresa. Dirección y gestión. España: Alfaomega.

[3] Eisenhardt, K. (1989). Building Theories from Case Study Research. Academy of Management Review, 14(4), 532-550.

[4] Fernández Sánchez, E. (2005). Estrategia de inovación. Argentina: Paraninfo.

[5] Freitas-Filho, F.L. (2013). Gestão da inovação. Teoria e pratica para implantação. Brasil: Atlas S.A.

[6] Krušinskas, R.; Benetytė, R. (2015). Management Problems of Investment in Technological Innovation, Using Artificial Neural Network. Procedia - Social and Behavioral Sciences, 213, 442-447.

[7] Nowacki, R.; Bachnik, K. (2016). Innovations within knowledge management. Journal of Business Research, 69(5), 1577-1581.

[8] Pacelli, L. (2004). The Project Management Advisor: 18 major project screw-ups, and how to cut them off at the pass. Pearson Education.

[9] Quiroga, O. y Borrás, M.A. (septiembre, 2015). Metodologia de pesquisa qualitativa para estimular à inovação tecnológica. Trabajo presentado en el XV Simpósio de Engenharia da Produção Sul Americano (XV SEPROSUL), Sorocaba, Brasil.

[10] Quiroga, O. y Rossetti, G. (2016). Modelo extendido a estimular la innovación de productos-procesos. Trabajo presentado en el Congreso de Ingeniería de Procesos y Productos (CIPP 2016), Rosario, Argentina.

[11] Secretaría de la Pequeña y Mediana Empresa y Desarrollo Regional. (2010). Micro, Pequeñas y Medianas Empresas. Resolución 21/2010. Artículo №1. Santa Fe, Argentina. 